TOKYo J. Math.

VoL. 5, No. 2, 1982

\title{
Examples of Simply Connected Compact Complex 3-folds
}

\author{
Masahide KATO \\ Sophia University
}

In this note, we shall construct a series of compact complex manifolds $\left\{M_{n}\right\}_{n=1,2,3}, \ldots$ of dimension 3 which are non-algebraic and nonkähler with the numerical characters $\pi_{1}\left(M_{n}\right)=0, \pi_{2}\left(M_{n}\right)=Z, b_{3}\left(M_{n}\right)=4 n$, $\operatorname{dim} H^{1}\left(M_{n}, \mathcal{O}\right) \geqq n$, and $\operatorname{dim} H^{1}\left(M_{n}, \Omega^{1}\right) \geqq n$, where $\Omega^{p}$ is the sheaf of germs of holomorphic $p$-forms. These examples show, in particular, that, it is impossible to estimate $h^{p, q}(M)=\operatorname{dim} H^{q}\left(M, \Omega^{p}\right)$ of a compact complex manifold $M$ in terms of its $(p+q)$-th Betti number, contrary to the case of dimension 2 or the case of kähler manifolds. To construct these examples, we employ a method of connecting two manifolds together to obtain a new one (see $\S \S 3$ and 4).

The discussions with Mr. H. Tsuji was very stimulating, to whom the author would like to express his hearty thanks.

$\S 1$. We shall construct, in this section, a complex manifold $X$ of dimension 3 with a projection

$$
p: X \longrightarrow C
$$

such that

(i) $X-p^{-1}(0)$ is biholomorphic to the product of a primary Hopf surface $S_{\alpha}=C^{2}-\{0\} /\langle\alpha\rangle$ and $C^{*}=C-\{0\}$ with $\alpha=\exp 2 \pi i a$;

(ii) $p^{-1}(0)$ is simply connected, and is a union of two primary Hopf surfaces biholomorphic to $S_{\beta j}=C^{2}-\{0\} /\left\langle\beta_{j}\right\rangle \quad(j=0,1)$ with $\beta_{j}=$ $\exp 2 \pi i b_{j}$ which intersect each other normally in an elliptic curve, where $a \in C$ is a fixed constant satisfying $\operatorname{Im} a>0, b_{0}=a^{-1}$, and $b_{1}=(1-a)^{-1}$. Let $a \in C$ be a fixed number such that $\operatorname{Im} a>0$. Then $\alpha=\exp 2 \pi i a$ satisfies $0<|\alpha|<1$. The multiplication $\xi \mapsto \alpha \xi$ for $\xi \in C^{*}=\{\xi \in C: \xi \neq 0\}$ defines a holomorphic automorphism of $C^{*}$ and the quotient space $C=$ $C^{*} \mid\langle\alpha\rangle$ is an elliptic curve. Denote by $[\xi]$ the point on $C$ corresponding to $\xi \in C^{*}$. Take three copies $W_{j}, j=1,2,3$, of $C^{2}$, on which we fix standard systems of coordinates $\left(x_{j}, y_{j}\right)$. Let $X_{j}=W_{j} \times C$, and let $\left(x_{j}, y_{j}\right.$ :

Received July 28, 1981 
$\left.\left[\xi_{j}\right]\right)$ be their coordinates. We form the complex 3-fold $X$ by patching $X_{j}$ 's as follows:

(1)

$$
\begin{aligned}
& X=\bigcup_{j=1}^{3} X_{j}, \\
& \left\{\begin{array}{l}
x_{2}=x_{1} y_{1} \\
y_{2}=x_{1}^{-1} \\
{\left[\xi_{2}\right]=\left[\xi_{1} x_{1}^{a}\right]}
\end{array} \quad \text { on } X_{1} \cap X_{2}, \quad\left\{\begin{array}{l}
x_{3}=x_{2} y_{2} \\
y_{3}=x_{2}^{-1} \\
{\left[\xi_{3}\right]=\left[\xi_{2} x_{2} x_{2}^{-a}\right]}
\end{array} \text { on } X_{2} \cap X_{3},\right.\right. \\
& \left\{\begin{array}{l}
x_{1}=x_{3}^{-1} y_{3}^{-1} \\
y_{1}=x_{3} \\
{\left[\xi_{1}\right]=\left[\xi_{3} x_{3}^{a} y_{8}\right]}
\end{array} \text { on } X_{3} \cap X_{1} .\right.
\end{aligned}
$$

It is easy to check that the patching is well-defined. Let $p$ be the holomorphic mapping of $X$ onto $C$ given by

$$
p= \begin{cases}y_{1} & \text { on } X_{1} \\ x_{2} y_{2} & \text { on } X_{2} \\ x_{3} & \text { on } X_{3} .\end{cases}
$$

We shall show that the fibre space

$$
p: X \longrightarrow C
$$

has the desired properties (i) and (ii), and see also some additional facts. Consider the following two 2 -folds $S_{0}$ and $S_{1}$ in $X$ :

$$
\begin{aligned}
& S_{0}: y_{1}=0 \text { in } X_{1}, \text { and } x_{2}=0 \text { in } X_{2} \\
& S_{1}: y_{2}=0 \text { in } X_{2}, \text { and } x_{3}=0 \text { in } X_{3},
\end{aligned}
$$

which are biholomorphic, respectively, to the primary Hopf surfaces

$$
S_{\beta_{j}}=C^{2}-\{0\} /\left\langle\beta_{j}\right\rangle, \quad j=0,1,
$$

where $\left\langle\beta_{j}\right\rangle$ is the infinite cyclic group generated by the holomorphic automorphism

$$
\begin{aligned}
\beta_{j}: C^{2}-\{0\} & \longrightarrow C^{2}-\{0\} \\
\Psi & \Psi \\
(x, y) & \longmapsto\left(\beta_{j} x, \beta_{j} y\right) .
\end{aligned}
$$

In fact, let

$$
\varphi_{01}: S_{0} \cap X_{1} \longrightarrow S_{\beta_{0}}
$$

be given by 
and let

$$
\left\{\begin{array}{l}
x=\xi_{1}^{a / 1} \\
y=x_{1} \xi_{1}^{1 / a}
\end{array}\right.
$$

be given by

$$
\varphi_{02}: S_{0} \cap X_{2} \longrightarrow S_{\beta_{0}}
$$

$$
\left\{\begin{array}{l}
x=y_{2} \xi_{2}^{1 / a} \\
y=\xi_{2}^{1 / a}
\end{array}\right.
$$

Then

$$
\varphi_{0}= \begin{cases}\varphi_{01} & \text { on } S_{0} \cap X_{1} \\ \varphi_{02} & \text { on } S_{0} \cap X_{2}\end{cases}
$$

gives a biholomorphic mapping of $S_{0}$ onto $S_{\beta_{0}}$. Similarly, let

$$
\psi_{12}: S_{1} \cap X_{2} \longrightarrow S_{\beta_{1}}
$$

be given by

$$
\left\{\begin{array}{l}
x=\xi_{2}^{1 /(1-a)} \\
y=x_{2} \xi_{2}^{1 /(1-a)}
\end{array}\right.
$$

and let

be given by

$$
\psi_{13}: S_{1} \cap X_{3} \longrightarrow S_{\beta_{1}}
$$

$$
\left\{\begin{array}{l}
x=y_{3} \xi_{3}^{1 /(1-a)} \\
y=\xi_{3}^{1 /(1-a)}
\end{array}\right.
$$

Then

$$
\psi_{1}= \begin{cases}\psi_{12} & \text { on } S_{1} \cap X_{2} \\ \psi_{13} & \text { on } S_{1} \cap X_{3}\end{cases}
$$

gives a biholomorphic mapping of $S_{1}$ onto $S_{\beta_{1}}$. By (2) and (3), we see that $p^{-1}(0)=S_{0} \cup S_{1}$. Since $S_{0} \cap S_{1}$ is in $X_{2}$ and $S_{0} \cup S_{1}$ is given in $X_{2}$ by $x_{2} y_{2}=0, S_{0}$ and $S_{1}$ intersect with each other normally in the elliptic curve

$$
\left\{\left(x_{2}, y_{2}:\left[\xi_{2}\right]\right): x_{2}=y_{2}=0\right\} \text {, }
$$

which is biholomorphic to $C^{*} /\langle\alpha\rangle$. Note that $[\xi] \mapsto\left[\xi^{b_{j}}\right]$ gives a biholomorphic 'map of $C^{*} /\langle\alpha\rangle$ to $C^{*} /\left\langle\beta_{j}\right\rangle$. Thus we see that $X$ has the property (ii) when we show the following.

Proposition 1. $p^{-1}(0)$ is simply connected. 
Proof. Since $\pi_{1}\left(p^{-1}(0)\right)$ is generated by the elements of $\pi_{1}\left(S_{0} \cap S_{1}\right) \cong$ $Z \oplus Z$, it is enough to show that each generator of $\pi_{1}\left(S_{0} \cap S_{1}\right)$ is nullhomotopic in $\pi_{1}\left(p^{-1}(0)\right)$. Let $s$ and $t$ be real numbers such that $0 \leqq s \leqq 1$, and $0 \leqq t \leqq 1$. Put

$$
\begin{aligned}
& \gamma_{s}:[0,1] \longrightarrow X_{2}, \quad \theta_{1} \in[0,1], \\
&\left\{\begin{array}{l}
x_{2}=0 \\
y_{2}=s e^{-2 \pi i \theta_{1}} \\
{\left[\xi_{2}\right]=\left[e^{2 \pi i a \theta_{1}}\right]}
\end{array}\right.
\end{aligned}
$$

and

$$
\begin{aligned}
\delta_{t}:[0,1] & \longrightarrow X_{2}, \quad \theta_{2} \in[0,1], \\
& \left\{\begin{array}{l}
x_{2}=t e^{-2 \pi i \theta_{2}} \\
y_{2}=0 \\
{\left[\xi_{2}\right]=\left[e^{2 \pi i(1-a) \theta_{2}}\right] .}
\end{array}\right.
\end{aligned}
$$

Then we see easily the following:

$$
\begin{aligned}
& \gamma_{s}([0,1]) \subset S_{0} \text { for all } s \text {, and } \gamma_{0}([0,1]) \subset S_{0} \cap S_{1}, \\
& \delta_{t}([0,1]) \subset S_{1} \text { for all } t \text {, and } \delta_{0}([0,1]) \subset S_{0} \cap S_{1}
\end{aligned}
$$

Moreover $\gamma_{0}$ and $\delta_{0}$ generate $\pi_{1}\left(S_{0} \cap S_{1}\right)$. To prove the proposition, it is enough to show that $\gamma_{1}$ is null-homotopic in $S_{0}$, and that $\delta_{1}$ is null-homotopic in $S_{1}$. By (1), $\gamma_{1}$ is given in $X_{1}$ by

$$
\left\{\begin{array}{l}
x_{1}=e^{2 \pi i \theta_{1}} \\
y_{1}=0 \\
{\left[\xi_{1}\right]=[1] .}
\end{array}\right.
$$

Hence $\gamma_{1}$ is null-homotopic in $S_{0} \cap X_{1} \subset S_{0}$. Similarly, $\delta_{1}$ is given in $X_{3}$ by

$$
\left\{\begin{array}{l}
x_{3}=0 \\
y_{3}=e^{2 \pi i \theta_{2}} \\
{\left[\xi_{3}\right]=[1] .}
\end{array}\right.
$$

Hence $\delta_{1}$ is null-homotopic in $S_{1} \cap X_{3} \subset S_{1}$.

Q.E.D.

Let

$$
W=\bigcup_{j=1}^{8} W_{j}
$$

be the complex 2-fold defined by patching $W_{j}$ 's as follows: 


$$
\begin{aligned}
& \left\{\begin{array}{l}
x_{2}=x_{1} y_{1} \\
y_{2}=x_{1}^{-1}
\end{array} \text { on } W_{1} \cap W_{2}, \quad\left\{\begin{array}{l}
x_{3}=x_{2} y_{2} \\
y_{3}=x_{2}^{-1}
\end{array} \text { on } W_{2} \cap W_{3},\right.\right. \\
& \left\{\begin{array}{l}
x_{1}=x_{3}^{-1} y_{3}^{-1} \\
y_{1}=x_{3}
\end{array} \text { on } W_{3} \cap W_{1} .\right.
\end{aligned}
$$

Then the projections

$$
\left(x_{j}, y_{j}:\left[\xi_{j}\right]\right) \longmapsto\left(x_{j}, y_{j}\right)
$$

define a projection

$$
\pi_{X}: X \longrightarrow W .
$$

Note that $X$ becomes a complex analytic fibre bundle over $W$ with the fibre $C^{*} \mid\langle\alpha\rangle$ by means of this projection. Let $t$ be the holomorphic mapping of $W$ onto $C$ given by

$$
t=\left\{\begin{array}{ll}
y_{1} & \text { on } W_{1} \\
x_{2} y_{2} & \text { on } W_{2} \\
x_{3} & \text { on } W_{3}
\end{array} .\right.
$$

Then we have the commutative diagram of projections:

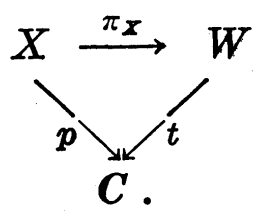

Take the primary Hopf surface

$$
S_{\alpha}=C^{2}-\{0\} /\langle\alpha\rangle,
$$

which is defined by identifying $(x, y) \in C^{2}-\{0\}$ with $(\alpha x, \alpha y) \in C^{2}-\{0\}$, where $\alpha=\exp 2 \pi i a$. Let $[x, y] \in S$ denote the point corresponding to $(x, y) \in C^{2}-\{0\} . \quad$ Put

$$
Y=S_{\alpha} \times C
$$

and consider the set

$$
E=\{([x, y], s) \in Y: y=s=0\},
$$

which is biholomorphic to $C^{*} /\langle\alpha\rangle$. Let

$$
q: Y \longrightarrow C
$$

be the projection to the 2 nd component. Take two copies $Z_{j}, j=1,2$, of $C^{2}$, and we form a complex 2-fold 


$$
Z=\bigcup_{j=1}^{2} Z_{j}
$$

as follows. Letting $\left(u_{j}, v_{j}\right)$ be a standard system of coordinates on $Z_{j}$, we identify $\left(u_{1}, v_{1}\right)$ with $\left(u_{2}, v_{2}\right)$, if and only if

$$
\left\{\begin{array}{l}
u_{1}=v_{2} \\
v_{1}=u_{2}^{-1} .
\end{array}\right.
$$

There is a holomorphic projection

$$
\pi_{Y}: Y \longrightarrow Z
$$

defined as follows. Let

$$
\begin{aligned}
& Y_{1}=\{([x, y], s) \in Y: x \neq 0\} \\
& Y_{2}=\{([x, y], s) \in Y: y \neq 0\}
\end{aligned}
$$

Then $\pi_{Y}$ is given by

$$
\begin{array}{ll}
\pi_{Y} \mid Y_{1}: u_{1}=s, & v_{1}=x^{-1} y, \\
\pi_{Y} \mid Y_{2}: u_{2}=x y^{-1}, & v_{2}=8 .
\end{array}
$$

Note that $Y$ becomes a complex analytic fibre bundle over $Z$ with the fibre $C^{*} \mid\langle\alpha\rangle$ by means of this projection. There is also a holomorphic mapping

given by

$$
\mu^{\prime}: W \longrightarrow Z
$$

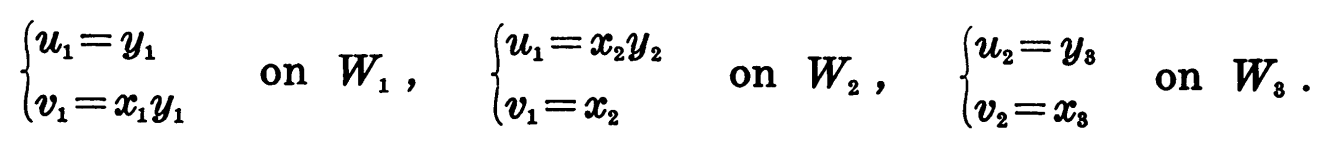

Then $\mu$ is the blowing-down of $W$ which contracts

$$
l=\left\{\left(x_{1}, y_{1}\right) \in W_{1}: y_{1}=0\right\} \cup\left\{\left(x_{2}, y_{2}\right) \in W_{2}: x_{2}=0\right\}
$$

to the point

$$
P=\left\{\left(u_{1}, v_{1}\right) \in Z_{1}: u_{1}=v_{1}=0\right\} .
$$

Now we shall prove the following proposition, from which the property (i) of $p: X \rightarrow C$ follows easily.

Proposition 2. There is a biholomorphic mapping

$$
\Psi^{\prime}: X-S_{0} \longrightarrow Y-E
$$

which makes the diagram 


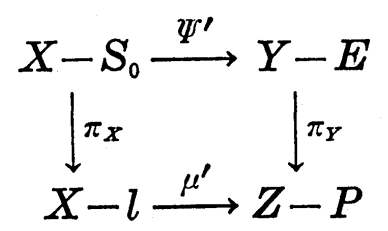

commutative.

Proof. Define $\Psi^{\prime}$ as follows:

$$
\begin{aligned}
& \Psi^{\prime} \mid X_{1}:\left\{\begin{array}{l}
x=\xi_{1} y_{1}^{-a} \\
y=\xi_{1} x_{1} y_{1} y_{1}^{-a} \\
s=y_{1},
\end{array}\right. \\
& \Psi^{\prime} \mid X_{2}:\left\{\begin{array}{l}
x=\xi_{2} x_{2}^{-a} \\
y=\xi_{2} x_{2} x_{2}^{-a} \\
s=x_{2} y_{2},
\end{array}\right. \\
& \Psi^{\prime} \mid X_{3}:\left\{\begin{array}{l}
x=\xi_{3} y_{3} \\
y=\xi_{3} \\
s=x_{3} .
\end{array}\right.
\end{aligned}
$$

It is easy to see that $\Psi^{\prime}$ is well-defined and gives the desired biholomorphic mapping.

§ 2. We shall construct a compact complex 3-fold $M_{1}$ with $\pi_{1}\left(M_{1}\right)=0$, $\pi_{2}\left(M_{1}\right)=Z$, and $b_{3}\left(M_{1}\right)=4$. Let $\widetilde{V}$ be the vector bundle of rank 2 defined by the Whitney sum $\mathscr{O}_{P_{1}}(1) \oplus \mathcal{O}_{P^{1}}(1)$ of two line bundles of degree 1 on $\boldsymbol{P}^{1}$. Take two copies $\widetilde{V}_{1}, \widetilde{V}_{2}$ of $\boldsymbol{C}^{3}$. Let $\left(\xi_{j}, \zeta_{j}, s_{j}\right)$ be a standard system of coordinates on $\widetilde{V}_{j}$. Then $\widetilde{V}$ is obtained by taking the union $\widetilde{V}_{1} \cup \widetilde{V}_{2}$ identifying $\left(\xi_{1}, \zeta_{1}, s_{1}\right)$ with $\left(\xi_{2}, \zeta_{2}, s_{2}\right)$, if and only if

$$
\left\{\begin{array}{l}
\xi_{1}=\xi_{2} s_{2}^{-1} \\
\zeta_{1}=\zeta_{2} s_{2}^{-1} \\
s_{1}=s_{2}^{-1} .
\end{array}\right.
$$

Put $l_{0}=\left\{\xi_{1}=\zeta_{1}=0\right\} \cup\left\{\xi_{2}=\zeta_{2}=0\right\}$ and $\widetilde{V}^{*}=\tilde{V}-l_{0}$. Let $\alpha$ be a holomorphic automorphism of $\tilde{V}^{*}$ defined by

$$
\left(\xi_{j}, \zeta_{j}, s_{j}\right) \longmapsto\left(\alpha \xi_{j}, \alpha \zeta_{j}, s_{j}\right) \quad \text { on } \tilde{V}^{*} \cap \widetilde{V}_{j},
$$

$j=1,2$. Put

$$
M=\widetilde{V}^{*} /\langle\alpha\rangle .
$$

Then the canonical projection $\tilde{\pi}: \tilde{V} \rightarrow \boldsymbol{P}^{1}$ induces a projection 
$\pi: M \longrightarrow P^{1}$

and define a structure on $M$ of a complex analytic fibre bundle over $\boldsymbol{P}^{1}$ with the fibre $S_{\alpha}$. Now we shall modify $M$ to obtain $M_{1}$. Put $V_{j}=$ $\left(\tilde{V}_{j} \cap \tilde{V}\right) /\langle\alpha\rangle(j=1,2)$. Obviously, $V_{j}, j=1,2$, are subdomains in $M$, and $M=V_{1} \cup V_{2}$. We replace $V_{1}$ by $X$ constructed in $\S 1$ as follows. Let

$$
\Phi_{1}: V_{1} \longrightarrow Y=S_{\alpha} \times C
$$

be the natural isomorphism induced by

$$
\left(\xi_{1}, \zeta_{1}, s_{1}\right) \longmapsto\left(\left[\xi_{1}, \zeta_{1}\right], s_{1}\right) .
$$

We have another isomorphism

$$
\Phi: X-p^{-1}(0) \longrightarrow S_{\alpha} \times C^{*} \subset Y,
$$

which is given by

$$
\Phi=\Psi^{\prime} \mid\left(X-p^{-1}(0)\right) .
$$

Therefore we can define a compact complex 3-fold

$$
M_{1}=X \cup V_{2}
$$

by identifying $x \in V_{1}-\pi^{-1}(0)=V_{1} \cap V_{2}$ with $\Phi^{-1} \circ \Phi_{1}(x) \in X-p^{-1}(0)$, where $0 \in P^{1}$ indicates the point $s_{1}=0$. Then $M_{1}$ is a complex analytic fibre space over $P^{1}$ with the projection

$$
p_{1}=\left\{\begin{array}{ll}
p & \text { on } X \\
\pi & \text { on } V_{2}
\end{array} .\right.
$$

Note that, for $s \in P^{1}, s \neq 0, p_{1}^{-1}(s)$ is biholomorphic to $S_{\alpha}$ and $p_{1}^{-1}(0)$ is biholomorphic to $S_{0} \cup S_{1}$.

Proposition 3.

(i) $\pi_{1}\left(M_{1}\right)=0$,

(ii) $\pi_{2}\left(M_{1}\right)=Z$,

(iii) $b_{3}\left(M_{1}\right)=4$, in particular, the Euler number $\theta\left(M_{1}\right)=0$.

Proof. (i) It is clear that

$$
i_{*}: \pi_{1}\left(M-\pi^{-1}(0)\right) \longrightarrow \pi_{1}\left(M_{1}\right)
$$

is surjective, where $i_{*}$ is induced by the natural inclusion. Note that $\pi_{1}\left(M-\pi^{-1}(0)\right) \cong Z$ is generated by a closed path contained in a fibre of $\pi$. Since $p_{1}^{-1}(0)$ is simply connected by Proposition 1 , we infer that $i_{*}$ 
is a zero mapping. Hence $\pi_{1}\left(M_{1}\right)=0$. (ii) Since $\pi_{1}\left(M_{1}\right)=0$, it is enough to show that $H_{2}\left(M_{1}, Z\right)=Z$ by the Hurewicz isomorphism theorem. Let $\Delta$ be a small disc around $0 \in \boldsymbol{P}^{1}$. Then we have the Mayer-Vietoris sequence with $Z$-coefficients:

$$
\begin{aligned}
\cdots \longrightarrow H_{2}\left(M_{1}-p_{1}^{-1}(0)\right) & \oplus H_{2}\left(p_{1}^{-1}(\Delta)\right) \stackrel{q_{2}}{\longrightarrow} H_{2}\left(M_{1}\right) \longrightarrow H_{1}\left(p_{1}^{-1}(\partial \Delta)\right) \\
& \stackrel{i_{1} \oplus j_{1}}{\longrightarrow} H_{1}\left(M_{1}-p_{1}^{-1}(0)\right) \oplus H_{1}\left(p_{1}^{-1}(\Delta)\right) \longrightarrow \cdots .
\end{aligned}
$$

First we claim that $H_{2}\left(p_{1}^{-1}(\Delta)\right)=0$. Since $p_{1}^{-1}(0)=p^{-1}(0)$ is a deformation retract of $p_{1}^{-1}(\Delta)$, it is enough to show that $H_{2}\left(p^{-1}(0)\right)=0$. Recall that $p^{-1}(0)=S_{0} \cup S_{1}$. We consider the Mayer-Vietoris sequence with $Z$-coefficients

$$
\left.\cdots \longrightarrow H_{2}\left(S_{0}\right) \oplus H_{2}\left(S_{1}\right) \longrightarrow H_{2}\left(p^{-1}(0)\right) \underset{H}{\longrightarrow} H_{1}\left(S_{0}\right) \oplus H_{2}\left(S_{1}\right) \longrightarrow S_{1}\right) \longrightarrow .
$$

By the argument in the proof of Proposition 1, we see that

$$
H_{1}\left(S_{0} \cap S_{1}\right) \longrightarrow H_{1}\left(S_{0}\right) \oplus H_{1}\left(S_{1}\right)
$$

is bijective. Moreover, it is clear that $H_{2}\left(S_{j}\right)=0, j=0,1$. Therefore we have $H_{2}\left(p^{-1}(0)\right)=0$. Next we claim that the kernel of

$$
i_{1}: H_{1}\left(p_{1}^{-1}(\partial \Delta)\right) \longrightarrow H_{1}\left(M_{1}-p_{1}^{-1}(0)\right)
$$

is isomorphic to $\boldsymbol{Z}$. Note that, by Proposition 2,

$$
p_{1}^{-1}(\partial \Delta) \cong S^{1} \times S_{\alpha} \cong S^{1} \times S^{1} \times S^{3}
$$

and

$$
M_{1}-p_{1}^{-1}(0) \cong M-\pi^{-1}(0) \cong C \times S_{\alpha} \cong R^{2} \times S^{1} \times S^{s} .
$$

Therefore the 1-cycle $\gamma_{b}$ in $p_{1}^{-1}(\partial \Delta)$ defined by $S^{1} \times\{q\}, q \in S_{\alpha}$, is a free basis of the kernel of $i_{1}$. Hence $H_{2}\left(M_{1}\right)=Z$ follows from (5) and (4). (iii) Since the Euler number $e\left(M_{1}\right)$ is equal to that of $M$, we have $b_{3}\left(M_{1}\right)=4$.

Q.E.D.

Take two copies $Z^{\prime}, Z^{\prime \prime}$ of $Z$. Let $\left(u_{j}^{\prime}, v_{j}^{\prime}\right)$ (resp. $\left.\left(u_{j}^{\prime \prime}, v_{j}^{\prime \prime}\right)\right)$ be the local coordinates on $Z^{\prime}$ (resp. $Z^{\prime \prime}$ ) corresponding to $\left(u_{j}, v_{j}\right)$ on $Z$. We form the union

$$
R_{1}=Z^{\prime} \cup W
$$

by the identifications: 


$$
\begin{array}{ll}
\left(u_{1}^{\prime}, v_{1}^{\prime}\right)=\left(x_{1}, y_{1}\right) \text { iff } x_{1}=u_{1}^{\prime} v_{1}^{\prime}, & y_{1}=u_{1}^{\prime-1}, \\
\left(u_{1}^{\prime}, v_{1}^{\prime}\right)=\left(x_{2}, y_{2}\right) \text { iff } x_{2}=v_{1}^{\prime}, & y_{2}=u_{1}^{\prime-1} v_{1}^{\prime-1}, \\
\left(u_{1}^{\prime}, v_{1}^{\prime}\right)=\left(x_{3}, y_{3}\right) \text { iff } x_{3}=u_{1}^{\prime-1}, & y_{3}=v_{1}^{\prime-1}, \\
\left(u_{2}^{\prime}, v_{2}^{\prime}\right)=\left(x_{1}, y_{1}\right) \text { iff } x_{1}=u_{2}^{\prime-1} v_{2}^{\prime}, & y_{1}=v_{2}^{\prime-1}, \\
\left(u_{2}^{\prime}, v_{2}^{\prime}\right)=\left(x_{2}, y_{2}\right) \text { iff } x_{2}=u_{2}^{\prime-1}, & y_{2}=u_{2}^{\prime} v_{2}^{\prime-1}, \\
\left(u_{2}^{\prime}, v_{2}^{\prime}\right)=\left(x_{3}, y_{3}\right) \text { iff } x_{3}=v_{2}^{\prime-1}, & y_{3}=u_{2}^{\prime} .
\end{array}
$$

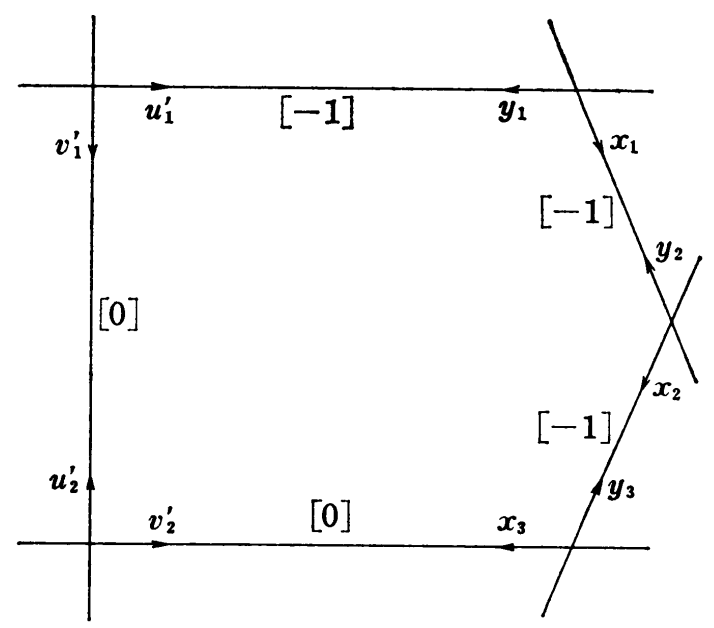

[ ] indicates the self-intersection number of a curve.

$$
\text { Figure } R_{1}
$$

Let

$$
\pi_{V_{2}}: V_{2} \longrightarrow Z^{\prime}
$$

be the holomorphic mapping given by

$$
\begin{aligned}
& \left\{\begin{array}{l}
u_{1}^{\prime}=s_{2} \\
v_{1}^{\prime}=\zeta_{2} \xi_{2}^{-1}
\end{array} \text { if } \xi_{2} \neq 0, \quad\right. \text { and } \\
& \left\{\begin{array}{l}
v_{2}^{\prime}=s_{2} \\
u_{2}^{\prime}=\zeta_{2}^{-1} \xi_{2}
\end{array} \quad \text { if } \zeta_{2} \neq 0 .\right.
\end{aligned}
$$

Define

$$
\pi_{M_{1}}: M_{1} \longrightarrow R_{1}
$$

by

$$
\pi_{M_{1}}= \begin{cases}\pi_{X} & \text { on } X \\ \pi_{V_{2}} & \text { on } V_{2} .\end{cases}
$$

Then $M_{1}$ is a complex analytic fibre bundle over $R_{1}$ with the fibre 
$C^{*} \mid\langle\alpha\rangle$ and with the projection $\pi_{M_{1}}$. Similarly we form the union

$$
R=Z^{\prime \prime} \cup Z
$$

by the identifications:

$$
\begin{aligned}
& \left(u_{1}^{\prime \prime}, v_{1}^{\prime \prime}\right)=\left(u_{1}, v_{1}\right) \text { iff } u_{1}^{\prime \prime} u_{1}=1, \quad v_{1}^{\prime \prime}=v_{1}, \\
& \left(u_{1}^{\prime \prime}, v_{1}^{\prime \prime}\right)=\left(u_{2}, v_{2}\right) \text { iff } u_{1}^{\prime \prime} u_{2}=1, \quad v_{1}^{\prime \prime} u_{2}=1 \\
& \left(u_{2}^{\prime \prime}, v_{2}^{\prime \prime}\right)=\left(u_{1}, v_{1}\right) \text { iff } u_{2}^{\prime \prime} v_{1}=1, \quad v_{2}^{\prime \prime} u_{1}=1 \\
& \left(u_{2}^{\prime \prime}, v_{2}^{\prime \prime}\right)=\left(u_{2}, v_{2}\right) \text { iff } \\
& u_{2}^{\prime \prime}=u_{2}, \quad v_{2}^{\prime \prime} v_{2}=1
\end{aligned}
$$

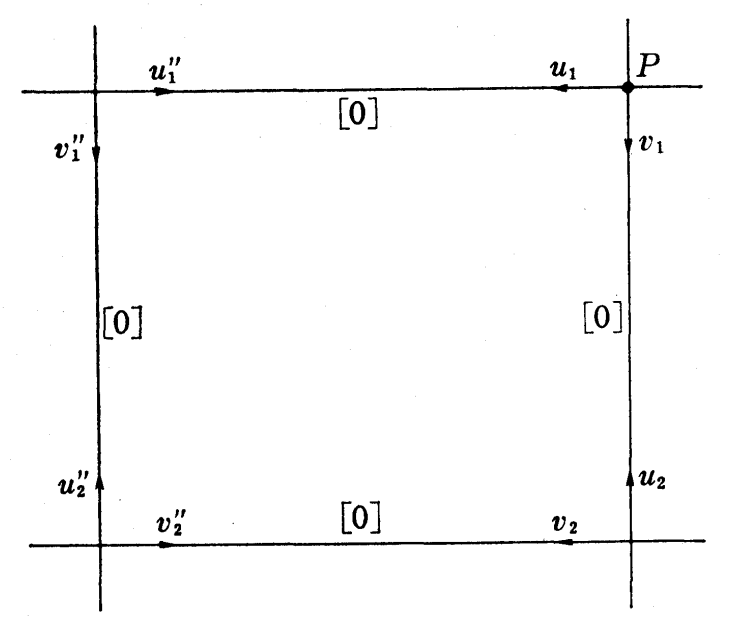

FiguRE $R$

Clearly, $R$ is biholomorphic to $\boldsymbol{P}^{1} \times \boldsymbol{P}^{1}$ and $R_{1}$ is the blowing-up of $R$ at $P=\left\{u_{1}=v_{1}=0\right\}$. Let $\mu: R_{1} \rightarrow R$ be the blowing-up. There is a projection

$$
\pi_{M}: M \longrightarrow R
$$

given by

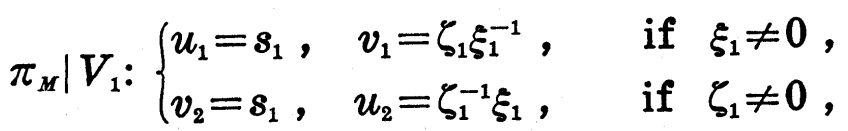

$$
\begin{aligned}
& \pi_{M} \mid V_{2}: \begin{cases}u_{1}^{\prime \prime}=s_{2}, v_{1}^{\prime \prime}=\zeta_{2} \xi_{2}^{-1}, & \text { if } \xi_{2} \neq 0, \\
v_{2}^{\prime \prime}=s_{2}, u_{2}^{\prime \prime}=\zeta_{2}^{-1} \xi_{2}, & \text { if } \zeta_{2} \neq 0 .\end{cases}
\end{aligned}
$$

The following proposition is clear from the above construction.

Proposition 4. The biholomorphic mapping

$$
\Psi^{\prime}: X-S_{0} \longrightarrow Y-E
$$

of Proposition 2 extends naturally to a biholomorphic mapping 


$$
\Psi: M_{1}-S_{0} \longrightarrow M-E,
$$

which makes the diagram

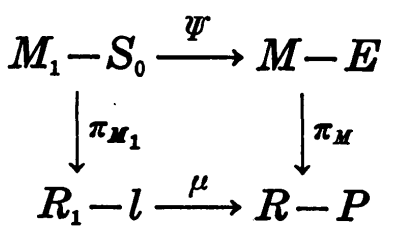

commutative.

PROPOSITION 5. There are non-singular rational curves $l_{q}$ in $M_{1}$, parametrized by $q=\left(\begin{array}{ll}q_{1} & r_{1} \\ q_{2} & r_{2}\end{array}\right) \in G L(2, C)$ with $q_{2} \neq 0$, such that each $l_{q}$ is a section of $p_{1}: M_{1} \rightarrow P^{1}$, and has a neighborhood isomorphic to that of a section of $\widetilde{V}=\mathcal{O}_{P^{1}}(1) \oplus \mathcal{O}_{P^{1}}(1)$.

PRoof. For each $q=\left(\begin{array}{ll}q_{1} & r_{1} \\ q_{2} & r_{2}\end{array}\right) \in G L(2, C)$ with $q_{2} \neq 0$, we define the section $\tilde{l}_{q}$ of $\tilde{V}^{*}=\tilde{V}-l_{0}$ by

$$
\left\{\begin{array} { l } 
{ \xi _ { 1 } = q _ { 1 } + r _ { 1 } s _ { 1 } } \\
{ \zeta _ { 1 } = q _ { 2 } + r _ { 2 } s _ { 1 } }
\end{array} \text { on } \widetilde { V } _ { 1 } , \text { and } \left\{\begin{array}{l}
\xi_{2}=q_{1} s_{2}+r_{1} \\
\zeta_{2}=q_{2} s_{2}+r_{2}
\end{array} \text { on } \widetilde{V}_{2}\right.\right. \text {. }
$$

Then the image $l_{q}^{\prime}$ of $\tilde{l}_{q}$ in $M$ does not intersect with $E$, and has a neighborhood in $M-E$ which is biholomorphic to a section of $\tilde{V}$. Put $l_{q}=\Psi^{-1}\left(l_{q}^{\prime}\right)$. Then the proposition follows from Proposition 4. Q.E.D.

$\S 3$. In this section we shall describe a method of connecting two compact complex 3-folds to obtain a new compact complex 3-fold. Let $\boldsymbol{P}^{3}$ be a complex projective space of dimension 3 and $\left[z_{0}: z_{1}: z_{2}: z_{3}\right]$ be a system of homogeneous coordinates. We define a holomorphic involution

by

$$
\sigma: P^{3} \longrightarrow P^{3}
$$

$$
\sigma\left(\left[z_{0}: z_{1}: z_{2}: z_{3}\right]\right)=\left[z_{2}: z_{3}: z_{0}: z_{1}\right] \text {. }
$$

Let $l$ and $l_{\infty}$ be skew lines in $P^{3}$ given by

and

$$
l: z_{0}=z_{1}=0 \text {, }
$$

$$
l_{\infty}: z_{2}=z_{3}=0 \text {. }
$$

It is easy to check that $\sigma(l)=l_{\infty}$. For any $r>0$, and $\varepsilon>1$, we define the following subsets in $P^{s}$ : 


$$
\begin{aligned}
& U_{r}=\left\{\left[z_{0}: z_{1}: z_{2}: z_{3}\right] \in P^{3}:\left|z_{0}\right|^{2}+\left|z_{1}\right|^{2}<r\left(\left|z_{2}\right|^{2}+\left|z_{3}\right|^{2}\right)\right\}, \\
& U=U_{1}, \\
& N(\varepsilon)=U_{\varepsilon}-\left[U_{1 / \varepsilon}\right],
\end{aligned}
$$

and

$$
\begin{aligned}
\Sigma & =\partial U \\
& =\left\{\left[z_{0}: z_{1}: z_{2}: z_{3}\right] \in P^{3}:\left|z_{0}\right|^{2}+\left|z_{1}\right|^{2}=\left|z_{2}\right|^{2}+\left|z_{3}\right|^{2}\right\} .
\end{aligned}
$$

Then $U_{r}$ and $N(\varepsilon)$ are connected and open, and $\Sigma$ is a non-singular real hypersurface in $\boldsymbol{P}^{3}$. It is easy to show the following two lemmas.

LemMA 1. For any $r>0, U_{r}$ is biholomorphic to $U$, and $\lim _{r \rightarrow 0} U_{r}=l$.

LEMMA 2. For any $\varepsilon>1$, we have
(i ) $\Sigma \subset N(\varepsilon)$,
(ii) $\sigma(\Sigma)=\Sigma$,
(iii) $\sigma(N(\varepsilon))=N(\varepsilon)$, and
(iv) $\sigma(U)=P^{3}-[U]$.

A compact complex 3-fold $M$ is said to be of type Class $L$ if and only if $M$ contains a subdomain which is biholomorphic to $N(\varepsilon)$ for some $\varepsilon>1$.

Let

$$
F: \tilde{V} \longrightarrow P^{3}-l_{\infty}
$$

be the biholomorphic mapping defined by

$$
F \mid V_{1}:\left(\xi_{1}, \zeta_{1}, s_{1}\right) \longmapsto\left[\xi_{1}: \zeta_{1}: s_{1}: 1\right]
$$

and

$$
F \mid V_{2}:\left(\xi_{2}, \zeta_{2}, s_{2}\right) \longmapsto\left[\xi_{2}: \zeta_{2}: 1: s_{2}\right]
$$

From this we have

LEMMA 3. Each section of $\widetilde{V}$ is mapped by $F$ to a projective line in $\boldsymbol{P}^{8}$ outside $l_{\infty}$.

For any $\varepsilon>1, N(\varepsilon)$ contains (infinitely many) projective lines in $\boldsymbol{P}^{3}$. Therefore, by Lemma 1, we have

Lemma 4. Suppose that $M$ is of Class $L$. Then there is a nonsingular rational curve $C$ and its neighborhood in $M$ which is biholomorphic to $U_{\varepsilon}$ for some $\varepsilon>1$.

Suppose that $M_{1}$ and $M_{2}$ are of Class $L$. For some $\varepsilon>1$, there are open embeddings 


$$
i_{\nu}: U_{\iota} \longrightarrow M_{\nu}(\nu=1,2)
$$

Let

$$
M_{\nu}^{*}=M_{\nu}-\left[i_{\nu}\left(U_{1 / 8}\right)\right]
$$

and form the union

$$
M_{1}^{*} \cup M_{2}^{*}
$$

by identifying a point $x_{1} \in i_{1}(N(\varepsilon)) \subset M_{1}^{*}$ with the point $x_{2}=i_{2} \circ \sigma \circ i_{1}^{-1}\left(x_{1}\right) \in M_{2}^{*}$.

LEMMA 5. $M_{1}^{*} \cup M_{2}^{*}$ is a compact complex 3-fold.

Proof is easy.

REMARK 1. If $M_{1}=M_{2}=P^{3}$ and $i_{\nu}$ are the natural inclusions, then $M_{1}^{*} \cup M_{2}^{*}=P^{3}$.

We denote $M_{1}^{*} \cup M_{2}^{*}$ by $M\left(M_{1}, M_{2}, i_{1}, i_{2}\right)$. It is clear that $M\left(M_{1}, M_{2}\right.$, $\left.i_{1}, i_{2}\right)$ is defined independently of the choice of $\varepsilon$, but may depend on the choice of $i_{\nu}$ 's. The process to construct $M\left(M_{1}, M_{2}, i_{1}, i_{2}\right)$ out of $M_{\nu}$ 's and $i_{\nu}$ 's is called a connecting operation. Note that $M\left(M_{1}, M_{2}, i_{1}, i_{2}\right)$ is also of Class $L$.

§4. By means of connecting operations, we shall construct inductively a series of compact complex 3 -folds $\left\{M_{n}\right\}_{n=1,2,3}, \ldots$ stated in the beginning of this note. Let $M_{1}$ be the manifold constructed in $\$ 2$, which is of Class $L$ by Proposition 5. To construct $M_{2}$, we take two copies of $M_{1}$, say $M_{1}$ and $M_{1}^{\prime}$. In the following, $A^{\prime}$ indicates a subset in $M_{1}^{\prime}$ corresponding to $A$ in $M_{1}$. Let $l_{q_{1}}$ (resp. $l_{q_{1}}^{\prime}$ ) be one of the nonsingular rational curves in $M_{1}$ (resp. $M_{1}^{\prime}$ ) described in Proposition 5 . Let $L_{1}$ (resp. $L_{1}^{\prime}$ ) be a neighborhood of $l_{q_{1}}$ (resp. $l_{q_{1}}^{\prime}$ ) in $M_{1}-S_{0}$ (resp. $M_{1}^{\prime}-$ $S_{0}^{\prime}$ ) which is biholomorphic to $U_{t_{1}}$ for some $\varepsilon_{1}>1$. This is possible by Lemma 1. Let $i_{1}: U_{i_{1}} \rightarrow L_{1} \subset M_{1}$ (resp. $i_{1}^{\prime}: U_{i_{1}} \rightarrow L_{1}^{\prime} \subset M_{1}^{\prime}$ ) be an isomorphism. By the connecting operation, we obtain a compact complex 3-fold

$$
M_{2}=M\left(M_{1}, M_{1}^{\prime}, i_{1}, i_{1}^{\prime}\right) \text {. }
$$

Note that $M_{2}$ contains at least two Hopf surfaces $H_{1}$ and $H_{2}$, corresponding to $S_{0}$ and $S_{0}^{\prime}$ in $M_{1}$ and $M_{1}^{\prime}$, respectively. Now we regard $i_{1}\left(N\left(\varepsilon_{1}\right)\right.$ ) as a subdomain in $M_{2}$. In $i_{1}\left(N\left(\varepsilon_{1}\right)\right)$, there are a non-singular rational curve $l_{q_{2}}$ and its neighborhood $L_{2}$ which is biholomorphic to that of a section of $\widetilde{V}$. Let $i_{2}: U_{\varepsilon_{2}} \rightarrow L_{2}\left(\subset i_{1}\left(N\left(\varepsilon_{1}\right)\right) \subset M_{2}\right)$ be an isomorphism, where we can assume that $1<\varepsilon_{2} \leqq \varepsilon_{1}$. By using $i_{1} \mid U_{\varepsilon_{2}}$ and $i_{2}$, we can connect $M_{1}$ and $M_{2}$, and obtain 


$$
M_{3}=M\left(M_{1}, M_{2}, i_{1} \mid U_{s_{2}}, i_{2}\right) .
$$

Since $i_{1}\left(N\left(\varepsilon_{1}\right)\right) \subset M_{2}-\left(H_{1} \cup H_{2}\right), M_{3}$ contains at least 3 Hopf surfaces $H_{1}$, $H_{2}$, and $H_{3}$ which correspond, respectively, to $H_{1}$ and $H_{2}$ in $M_{2}$, and $S_{0}$ in $M_{1}$. Now again, regarding $i_{1}\left(N\left(\varepsilon_{2}\right)\right)$ as a subdomain in $M_{3}$, we can repeat the above step, and we have inductively a series $\left\{M_{n}\right\}_{n=1,2}, \ldots$

$$
M_{n}=M\left(M_{1}, M_{n-1}, i_{1} \mid U_{e_{n-1}}, i_{n-1}\right)
$$

of compact complex 3 -folds. $M_{n}$ contains at least $n$ Hopf surfaces, one of which is from $M_{1}$ and the others are from $M_{n-1}$.

THEOREM. For all $n \geqq 1$,

(i) $M_{n}$ is non-algebraic and non-kähler,

(ii) $\pi_{1}\left(M_{n}\right)=0, \pi_{2}\left(M_{n}\right)=Z$, and $b_{3}\left(M_{n}\right)=4 n$,

(iii) $\operatorname{dim} H^{1}\left(M_{n}, \mathcal{O}\right) \geqq n$,

(iv) $\operatorname{dim} H^{1}\left(M_{n}, \Omega^{1}\right) \geqq n$.

Proof. (i) is clear, since $M_{n}$ contains Hopf surfaces. (ii) By the Mayer-Vietoris sequence with $Z$-coefficients

$$
\begin{aligned}
\cdots \longrightarrow H_{2}\left(M_{1}^{n-1} \cap M_{n-1}^{\sharp}\right) \stackrel{i_{2} \oplus j_{2}}{\longrightarrow} & H_{2}\left(M_{1}^{n-1}\right) \bigoplus H_{2}\left(M_{n-1}^{\sharp}\right) \longrightarrow H_{2}\left(M_{n}\right) \\
\longrightarrow & \longrightarrow H_{1}\left(M_{1}^{n-1} \cap M_{n-1}^{\sharp}\right)
\end{aligned}
$$

where

$$
M_{1}^{n-1}=M_{1}-\left[i_{1}\left(U_{1 / s_{n-1}}\right)\right],
$$

and

$$
M_{n-1}^{\sharp}=M_{n-1}-\left[i_{n-1}\left(U_{1 / s_{n-1}}\right)\right],
$$

we have

$$
H_{1}\left(M_{1}^{n-1} \cap M_{n-1}^{\sharp}\right)=0,
$$

and

$$
H_{2}\left(M_{1}^{n-1} \cap M_{n-1}^{\sharp}\right)=Z \text {, }
$$

since $M_{1}^{n-1} \cap M_{n-1}^{\#}$ is homotopy equivalent to $S^{2} \times S^{8}$. Note that $l_{q_{n}}$ generates both $H_{2}\left(M_{1}^{n-1} \cap M_{n-1}^{\sharp}\right)$ and $H_{2}\left(M_{1}^{n-1}\right)$. Hence

$$
i_{2}: H_{2}\left(M_{1}^{n-1} \cap M_{n-1}^{\sharp}\right) \longrightarrow H_{2}\left(M_{1}^{n-1}\right)
$$

is bijective. Therefore, from (6), we have

$$
H_{2}\left(M_{n}\right)=H_{2}\left(M_{n-1}^{*}\right) \text {. }
$$

By the exact sequence 


$$
\begin{aligned}
\cdots \longrightarrow H_{3}\left(M_{n-1}, M_{n-1}^{*}\right) \longrightarrow & H_{2}\left(M_{n-1}^{*}\right) \longrightarrow H_{2}\left(M_{n-1}\right) \\
& \longrightarrow H_{2}\left(M_{n-1}, M_{n-1}^{*}\right) \longrightarrow \cdots,
\end{aligned}
$$

and the duality

and

$$
H_{3}\left(M_{n-1}, M_{n-1}^{*}\right)=H^{3}\left(l_{q_{n-1}}\right)=0,
$$

we have

$$
H_{2}\left(M_{n-1}, M_{n-1}^{k}\right)=H^{4}\left(l_{q_{n-1}}\right)=0,
$$

$$
H_{2}\left(M_{n-1}^{\sharp}\right)=H_{2}\left(M_{n-1}\right) .
$$

Hence, by (7) and the induction assumption, we obtain

$$
H_{2}\left(M_{n}\right)=H_{2}\left(M_{n-1}\right)=Z \text {. }
$$

Since $\pi_{1}\left(M_{n}\right)=0$ is clear, $\pi_{2}\left(M_{n}\right)=Z$ follows from the Hurewicz isomorphism theorem. Since $e\left(M_{n}\right)=e\left(M_{n-1}\right)+e\left(M_{1}\right)-4=e\left(M_{n-1}\right)-4=-4(n-1)$ by the induction assumption and Proposition 3, we have $b_{3}\left(M_{n}\right)=2+2 b_{2}\left(M_{n}\right)-$ $e\left(M_{n}\right)=4 n$.

To prove (iii) of the theorem, we shall make some preparations. Recall that

$$
\begin{aligned}
& M_{n}^{*}=M_{n}-\left[i_{n}\left(U_{1 / e_{n}}\right)\right], \\
& M_{1}^{n}=M_{1}-\left[i_{1}\left(U_{1 / e_{n}}\right)\right],
\end{aligned}
$$

and that

$$
M_{n+1}=M_{n}^{*} \cup M_{1}^{n} .
$$

Let

$$
\begin{aligned}
& f_{n}^{1}: M_{n}^{\sharp} \longrightarrow M_{n+1}, \quad \text { and } \\
& f_{n}^{2}: M_{1}^{n} \longrightarrow M_{n+1}
\end{aligned}
$$

be the natural inclusions. Then we have

$$
s_{n}:=\left(f_{n}^{2} \circ i_{1}\right)\left|N\left(\varepsilon_{n}\right)=\left(f_{n}^{1} \circ i_{n} \circ \sigma\right)\right| N\left(\varepsilon_{n}\right)
$$

which defines an embedding

Let

$$
N\left(\varepsilon_{n}\right) \longrightarrow M_{n+1} \cdot
$$

$$
\begin{aligned}
& \rho_{n}: N\left(\varepsilon_{n}\right) \longrightarrow M_{n}^{*}, \\
& \sigma_{n}: M_{n}^{*} \longrightarrow M_{n}, \quad \text { and } \\
& \tau_{n}: N\left(\varepsilon_{n}\right) \longrightarrow M_{n}
\end{aligned}
$$


be the open embeddings defined, respectively, by

$$
\begin{aligned}
& \rho_{n}=\left(i_{n} \circ \sigma\right) \mid N\left(\varepsilon_{n}\right), \\
& \sigma_{n}=\text { the natural inclusion, and } \\
& \tau_{n}=\sigma_{n} \circ \rho_{n} .
\end{aligned}
$$

Let

$$
t_{n}: N\left(\varepsilon_{n+1}\right) \longrightarrow N\left(\varepsilon_{n}\right)
$$

be the open embedding defined by

$$
t_{n}=\boldsymbol{s}_{n}^{-1} \circ \tau_{n+1}=\boldsymbol{s}_{n}^{-1} \circ\left(i_{n+1} \circ \sigma \mid N\left(\varepsilon_{n+1}\right)\right)
$$

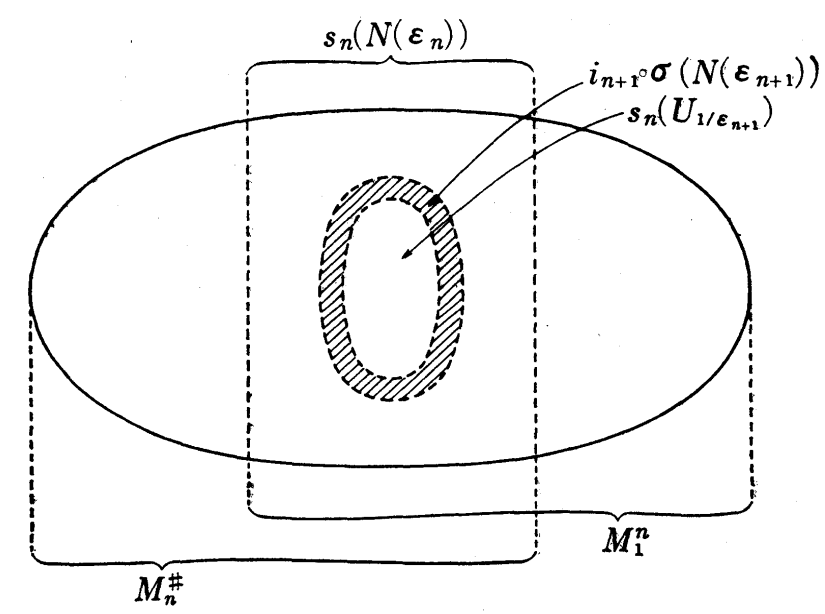

FIGURE $M_{n+1}$

Lemma 6. $\sigma_{n}^{*}: H^{1}\left(M_{n}, \mathcal{O}\right) \rightarrow H^{1}\left(M_{n}^{*}, \mathcal{O}\right)$ is injective for all $n \geqq 1$.

Proof. Since the homomorphism

$$
r_{1}: H^{1}\left(M_{n}-l_{q_{n}}, O\right) \longrightarrow H^{1}\left(M_{n}^{*}, \mathcal{O}\right)
$$

induced by the natural inclusion is injective by Andreotti-Siu [1, Proposition 1.2], it is enough to show that

$$
H_{l_{q_{n}}}^{1}\left(M_{n}, \mathcal{O}\right)=0 \text {. }
$$

Since $l_{q_{n}}$ has a neighborhood in $M_{n}$ which is biholomorphic to that of a projective line $\boldsymbol{P}^{1}$ in $\boldsymbol{P}^{3}$, we have the exact sequence

$$
\begin{aligned}
& \cdots \longrightarrow H^{0}\left(P^{3}-P^{1}, \mathcal{O}\right) \longrightarrow H^{0}\left(P^{3}, \mathcal{O}\right) \longrightarrow H_{l_{q_{n}}}^{1}\left(M_{n}, \mathcal{O}\right) \\
& \longrightarrow H^{1}\left(\boldsymbol{P}^{3}, \mathcal{O}\right) \longrightarrow \cdots
\end{aligned}
$$

From this sequence, (8) follows easily. $\quad$ Q.E.D. 
Let

$$
\begin{aligned}
& L_{1}=R^{1}\left(\pi_{M_{1}}\right)_{*} \mathcal{O}_{M_{1}}, \quad \text { and } \\
& L=R^{1}\left(\pi_{M}\right)_{*} \mathcal{O}_{M} .
\end{aligned}
$$

Then we have

LEMMA 7. $L_{1}=\mathcal{O}_{R_{1}}$, and $L=\mathcal{O}_{R}$.

Proof. First we consider $L_{1}$. By a theorem of Grauert, $L_{1}$ is a vector bundle of $\operatorname{rank} 1=\operatorname{dim} H^{1}\left(C^{*} \mid\langle\alpha\rangle, \mathcal{O}\right)$. Recall that $R_{1}$ is the blowing-up of $R, \mu: R_{1} \rightarrow R$, and that $R \cong P^{1} \times P^{1}$. Let $E_{1}$ be the proper inverse image of $P^{1} \times\{0\} \subset R$, and $E_{2}$ the proper inverse image of $\{0\} \times$ $P^{1} \subset R$. Then $H^{2}\left(R_{1}, Z\right)$ is generated by $E_{1}, E_{2}$, and the exceptional curve $l=\mu^{-1}(P)$. Note that $H^{1}\left(R_{1}, \mathcal{O}\right)=0$. Hence, to prove the lemma, it is enough to show that the restrictions of $L_{1}$ to $E_{1}, E_{2}$, and $l$ are trivial. But these are consequences of the fact that $\pi_{M_{1}}^{-1}\left(E_{1}\right), \pi_{M_{1}}^{-1}\left(E_{2}\right)$, and $\pi_{M_{1}}^{-1}(l)$ are all elliptic bundles with vanishing Chern numbers, by virtue of a result of Kodaira [3, Theorem 12]. By a similar argument, $L=\mathcal{O}_{R}$ can be proved easily.

LEMMA 8. $\operatorname{dim} H^{1}(M, \mathscr{O})=\operatorname{dim} H^{1}\left(M_{1}, \mathscr{O}\right)=1$.

Proof. This follows easily from Lemma 7 by using Leray's spectral sequences applied to the fibre bundles $\pi_{M}: M \rightarrow R$, and $\pi_{M_{1}}: M_{1} \rightarrow R_{1}$.

LEMMA 9. The homomorphism

$$
r_{2}: H^{1}\left(M_{1}, \mathcal{O}\right) \longrightarrow H^{1}\left(M_{0}-S_{0}, \mathcal{O}\right)
$$

induced by the natural inclusion is injective.

Proof. Since $L=\mathscr{O}_{R}$ by Lemma 7 , there is a non-zero section $s \in$ $H^{\circ}(R-P, L)$. By Proposition 4, we see that $\mu^{*} s \in H^{\circ}\left(R_{1}-l, L_{1}\right)$. Since $l$ is an exceptional curve in $R_{1}$, and since $L_{1}$ is trivial on $R_{1}$ by Lemma 7, $\mu^{*} s$ extends to a section $\widetilde{\mu^{*} s}$ of $H^{0}\left(R_{1}, L_{1}\right)$. Consider the commutative diagram

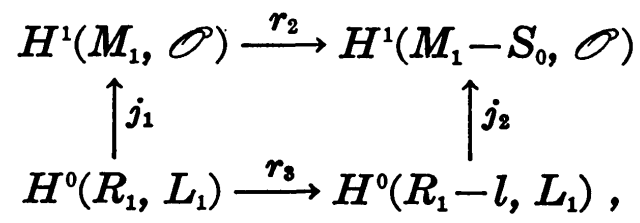

where $r_{3}$ is induced by the restrictions, and $j_{1}$ and $j_{2}$ are the canonical injections of Leray's spectral sequences. Then 


$$
r_{2} \circ j_{1}\left(\widetilde{\mu^{*} s}\right)=j_{2}\left(\mu^{*} s\right)
$$

Since $j_{2}$ is injective, and since $\mu^{*} s \neq 0$, we see that

$$
r_{2} \circ j_{1}\left(\widetilde{\mu^{*} s}\right) \neq 0 \text {. }
$$

By Lemmas 7 and 8, $j_{1}$ is an isomorphism. Therefore (10) implies that $r_{2}$ is injective.

Q.E.D.

LEMMA 10 . $\operatorname{dim} \operatorname{Ker} \rho_{1}^{*} \geqq 1$.

Proof. Consider the commutative diagram

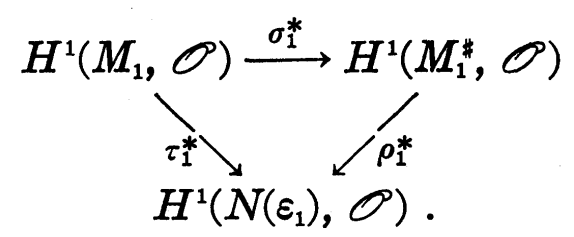

Take the element $j_{1}\left(\widetilde{\mu^{*} s}\right) \in H^{1}\left(M_{1}, \varnothing\right)$ of the proof of Lemma 9. By Lemma 6, $\sigma_{1}^{*} \circ j_{1}\left(\widetilde{\mu^{*} s}\right) \in H^{1}\left(M_{1}^{*}, \mathcal{O}\right)$ is not zero. Therefore, to prove the lemma, it suffices to show that

$$
\tau_{1}^{*} \circ j_{1}\left(\widetilde{\mu^{*} s}\right)=0 \text {. }
$$

The element $s \in H^{\circ}(R-P, L)$ extends to an element $\widetilde{s} \in H^{\circ}(R, L)$. Let $j_{3}: H^{0}(R, L) \rightarrow H^{1}(M, O)$ be the inclusion defined by Leray's spectral sequence. Consider the element $j_{3}(\widetilde{s}) \in H^{1}(M, \mathcal{O})$. Let

$$
\begin{aligned}
& \psi^{\prime}: H^{1}(M, C) \longrightarrow H^{1}\left(M_{1}-S_{0}, C\right), \quad \text { and } \\
& \psi^{\prime \prime}: H^{1}(M, \mathcal{O}) \longrightarrow H^{1}\left(M_{1}-S_{0}, \mathcal{O}\right)
\end{aligned}
$$

be the homomorphisms defined by the inclusion $M-E \rightarrow M$ followed by $\Psi^{-1}: M-E \rightarrow M_{1}-S_{0}$ of Proposition 4. Since $S_{0} \cap \tau_{1}\left(N\left(\varepsilon_{1}\right)\right)=\varnothing$, we have also the homomorphisms

$$
\begin{aligned}
& \tau_{1}^{\prime}: H^{1}\left(M_{1}-S_{0}, C\right) \longrightarrow H^{1}\left(N\left(\varepsilon_{1}\right), C\right), \text { and } \\
& \tau_{1}^{\prime \prime}: H^{1}\left(M_{1}-S_{0}, \mathcal{O}\right) \longrightarrow H^{1}\left(N\left(\varepsilon_{1}\right), \varnothing\right)
\end{aligned}
$$

induced by $\tau_{1}$. Then we have the following commutative diagram:

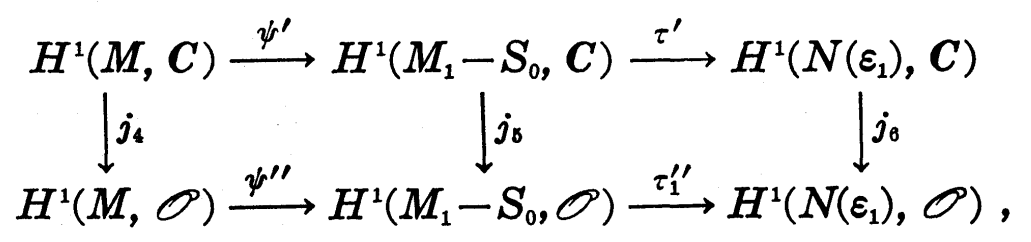


where $j_{4}, j_{8}$, and $j_{8}$ are homomorphisms defined by the natural inclusion $C \rightarrow \mathcal{O}$. It is easy to see that $\operatorname{dim} H^{0}(M, d \mathcal{O}) \leqq \operatorname{dim} H^{0}\left(M, \Omega^{1}\right)=0$, where $\Omega^{1}$ is the sheaf of germs of holomorphic 1-forms and $d \mathcal{O}$ is the subsheaf of $\Omega^{1}$ whose elements are $d$-closed. Moreover $H^{1}(M, C)=C$. Hence, by Lemma 8 and the exact sequence

$$
\mathrm{O} \longrightarrow \mathrm{C} \longrightarrow \mathrm{O} \longrightarrow \mathrm{d} O \mathrm{O} \text {, }
$$

we see that $j_{4}$ is an isomorphism. Hence, from the diagram (13) and the fact that $H^{1}\left(N\left(\varepsilon_{1}\right), C\right)=0$,

$$
\tau_{1}^{\prime \prime} \circ \psi^{\prime \prime} \circ j_{s}(\widetilde{s})=0
$$

follows. Consider the commutative diagram

$$
\begin{array}{cc}
H^{1}\left(M_{1}-S_{0}, \mathcal{O}\right) \stackrel{\psi^{\prime \prime}}{\longleftarrow} H^{1}(M, \mathscr{O}) & \uparrow j_{j_{3}} \\
\uparrow_{j_{2}} & \stackrel{\mu_{1}^{*}}{\longleftarrow} H^{0}(R, L),
\end{array}
$$

where $\mu_{1}^{*}$ is induced by the inclusion $R-P \rightarrow R$ followed by the isomorphism $\mu: R_{1}-l \rightarrow R-P$. Note that

$$
\mu^{*} s=\mu_{1}^{*} \widetilde{s} .
$$

Then, by the diagrams (9), (11), (13), and (15), we have

$$
\begin{aligned}
\tau_{1}^{*} \circ j_{1}\left(\widetilde{\mu^{*} s}\right) & =\tau_{1}^{\prime \prime} \circ r_{2} \circ j_{1}\left(\widetilde{\mu^{*} s}\right) \\
& =\tau_{1}^{\prime \prime} \circ j_{2} \circ r_{3}\left(\widetilde{\mu^{*} s}\right) \\
& =\tau_{1}^{\prime \prime} \circ j_{2}\left(\mu^{*} s\right) \\
& =\tau_{1}^{\prime \prime} \circ j_{2}\left(\mu_{1}^{*} \widetilde{s}\right) \\
& =\tau_{1}^{\prime \prime} \circ \psi^{\prime \prime} \circ j_{3}(\widetilde{s}),
\end{aligned}
$$

which is equal to zero by (14). Thus (12) is obtained. Q.E.D.

Proof of (iii) of THE Theorem. Consider the following inequalities:

$\left({ }^{*}\right)_{n}$

$\left({ }^{* *}\right)_{n}$ $\operatorname{dim} H^{1}\left(M_{n}, \mathcal{O}\right) \geqq n$,

$\operatorname{dim} \operatorname{Ker} \rho_{n}^{*} \geqq n$.

We shall prove, by induction on $n$, that $\left({ }^{*}\right)_{n}$ and $\left({ }^{* *}\right)_{n}$ hold for all $n \geqq 1$. By Lemmas 8 and 10, $\left({ }^{*}\right)_{1}$ and $\left({ }^{* *}\right)_{1}$ hold. Suppose that $\left({ }^{*}\right)_{n}$ and $\left({ }^{* *}\right)_{n}$ hold for some $n \geqq 1$. Consider the Mayer-Vietoris sequence 


$$
\begin{aligned}
& \cdots \longrightarrow H^{1}\left(M_{n+1}, \mathcal{O}\right) \stackrel{f_{n}^{*}}{\longrightarrow} H^{1}\left(M_{n}^{\sharp}, \mathcal{O}\right) \oplus H^{1}\left(M_{1}^{n}, \mathcal{O}\right) \\
& \stackrel{g_{n}^{*}}{\longrightarrow} H^{1}\left(N\left(\varepsilon_{n}\right), \mathcal{O}\right) \longrightarrow \cdots,
\end{aligned}
$$

where

$$
\begin{aligned}
& f_{n}^{*}=f_{n}^{1 *} \oplus f_{n}^{2 *}, \quad \text { and } \\
& g_{n}^{*}=\rho_{n}^{*}-\left(i_{1} \mid N\left(\varepsilon_{n}\right)\right)^{*} .
\end{aligned}
$$

There is the following commutative diagram:

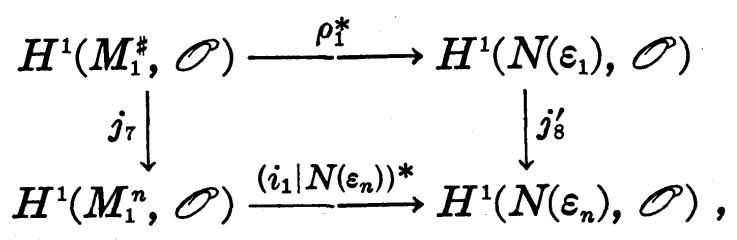

where $j_{7}$ is induced by the inclusion, and $j_{8}^{\prime}$ is induced by the inclusion followed by $\sigma$. Note that $j_{7}$ is injective by Andreotti-Siu [1, Proposition 1.2]. Hence by Lemma 10,

$$
1 \leqq \operatorname{dim} \operatorname{Ker} \rho_{1}^{*} \leqq \operatorname{dim} \operatorname{Ker}\left(i_{1} \mid N\left(\varepsilon_{n}\right)\right)^{*} .
$$

Since the subspace

$$
K:=\operatorname{Ker} \rho_{n}^{*} \oplus \operatorname{Ker}\left(i_{1} \mid N\left(\varepsilon_{n}\right)\right)^{*}
$$

in $H^{1}\left(M_{n}^{*}, \mathcal{O}\right) \oplus H^{1}\left(M_{1}^{n}, \mathcal{O}\right)$ is contained in $\operatorname{Ker} g_{n}^{*}$, we have

$$
\operatorname{dim} \operatorname{Ker} g_{n}^{*} \geqq n+1,
$$

by using (17) and the induction assumptions $\left({ }^{* *}\right)_{1}$ and $\left({ }^{* *}\right)_{n}$. Hence we obtain $\left({ }^{*}\right)_{n+1}$ by the exact sequence (16). Moreover, since

$$
f_{n}^{*-1}(K) \subset \operatorname{Ker} s_{n}^{*},
$$

we have

$$
\operatorname{dim} \operatorname{Ker} s_{n}^{*} \geqq \operatorname{dim} f_{n}^{*-1}(K) \geqq n+1 .
$$

Then by the commutative diagram

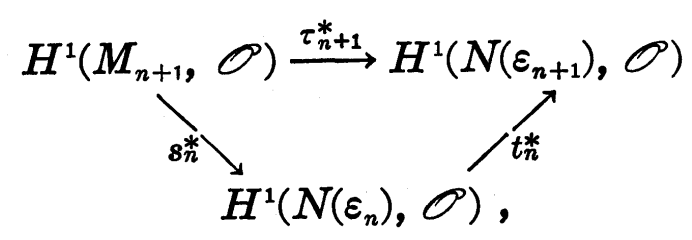

we obtain

$\operatorname{dim} \operatorname{Ker} \tau_{n+1}^{*} \geqq \operatorname{dim} \operatorname{Ker} s_{n}^{*} \geqq n+1$ 
Therefore, by the commutative diagram

and Lemma 6, we have

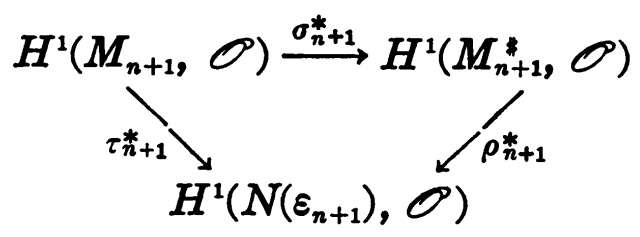

$$
\operatorname{dim} \operatorname{Ker} \rho_{n+1}^{*} \geqq \operatorname{dim} \operatorname{Ker} \tau_{n+1}^{*} \geqq n+1,
$$

which proves $\left({ }^{* *}\right)_{n+1}$.

Proof of (iv) of the Theorem. By the exact sequence

$$
\mathrm{O} \longrightarrow \mathrm{C} \longrightarrow \mathcal{O} \longrightarrow d \mathcal{O} \longrightarrow 0
$$

and $\pi_{1}\left(M_{n}\right)=0$, we have

$$
\operatorname{dim} H^{1}\left(M_{n}, \mathscr{O}\right) \leqq \operatorname{dim} H^{1}\left(M_{n}, d \mathscr{O}\right) .
$$

Letting $d \Omega^{1}$ be the subsheaf of $\Omega^{2}$ whose elements are $d$-closed, we form the exact sequence

$$
0 \longrightarrow d \mathcal{O} \longrightarrow \Omega^{1} \longrightarrow d \Omega^{1} \longrightarrow 0 \text {. }
$$

We claim that

$$
\operatorname{dim} H^{0}\left(M_{n}, d \Omega^{1}\right)=0 .
$$

To prove (20), it suffices to show that

$$
\operatorname{dim} H^{0}\left(M_{n}, \Omega^{2}\right)=0 \text {. }
$$

Take any $\omega \in H^{0}\left(M_{n}, \Omega^{2}\right)$. Then $i_{n}^{*} \omega \in H^{0}\left(U_{e_{n}}, \Omega^{2}\right)$. By Andreotti-Siu [1, Proposition 1.2], we have

$$
H^{0}\left(U_{s_{n}}, \Omega^{2}\right) \cong H^{0}\left(\boldsymbol{P}^{3}, \Omega^{2}\right)=0 .
$$

Hence $i_{n}^{*} \omega=0$. This implies $\omega=0$ and proves (21). Therefore, from (19) and (20),

$$
\operatorname{dim} H^{1}\left(M_{n}, d \mathcal{O}\right) \leqq \operatorname{dim} H^{1}\left(M_{n}, \Omega^{1}\right) .
$$

Thus combining this with (iii) and the inequality (18), we obtain

$$
\operatorname{dim} H^{1}\left(M_{n}, \Omega^{1}\right) \geqq n \text {. }
$$

REMARK 2.*' I don't know whether $\operatorname{dim} H^{1}\left(M_{n}, \mathcal{O}\right)=n$.

\footnotetext{
*) See the end of the paper.
} 
REMARK 3. For a compact complex manifold $X$, we put

$$
h^{p, q}(X)=\operatorname{dim} H^{q}\left(X, \Omega^{p}\right) \text {. }
$$

It is known that, if $X$ is a compact kähler manifold, or, more generally, a compact Fujiki manifold (i.e., of Class $\mathscr{C}$ in Fujiki [2, Definition 1.1]), then the equality

$$
h^{p, q}(X)=h^{q, p}(X)
$$

holds and the $k$-th Betti number is given by

$$
b_{k}(X)=\sum_{p+q=k} h^{p, q}(X) \text {. }
$$

Hence, in particular, we have

$$
h^{0,1}(X)=\frac{1}{2} b_{1}(X) \quad \text { and } \quad h^{1,1}(X) \leqq b_{2}(X) .
$$

By Kodaira [3, Theorem 3], we also see that, if $\operatorname{dim} X=2$, then the following equality and inequality hold including the cases where $X$ are non-kähler:

$$
\begin{aligned}
& h^{0,1}(X)= \begin{cases}\frac{1}{2} b_{1}(X), & \text { if } b_{1}(X) \equiv 0(\bmod 2) \\
\frac{1}{2}\left(b_{1}(X)+1\right), & \text { if } b_{1}(X) \equiv 1(\bmod 2),\end{cases} \\
& h^{1,1}(X) \leqq b_{2}(X) .
\end{aligned}
$$

Our example shows, however, that, for general compact complex manifolds of dimension more than 2 , it is impossible to estimate $h^{0,1}(X)$ and $h^{1,1}(X)$ in terms of $b_{1}(X)$ and $b_{2}(X)$, respectively.

REMARK 4. In his recent study of compact complex 3 -folds with Hopf surfaces as divisors, $\mathrm{H}$. Tsuji has also found a method of modifying a compact complex manifold as we have used in section $\S 2$. Namely, he found that, if a compact complex manifold $X, \operatorname{dim} X \geqq 3$, contains a primary Hopf manifold $S$ of codimension 1 with a certain condition on the normal bundle of $S$ in $X$, then one can replace $S$ by an elliptic curve $E$ to obtain a new compact complex manifold $Y=(X-S) \cup E$ [4].

Notes added on Dec. 10, 1981. It can be shown that $\operatorname{dim} H^{1}\left(M_{n}, \mathcal{O}\right)=$ $n$, and $\operatorname{dim} H^{2}\left(M_{n}, O^{\prime}\right)=0$. The differentiable structure of $M_{n}$ can be described completely by using connected sum operations by virtue of 
the results of C. T. C. Wall [Invent. Math., 1, 355-374 (1966)]. See the forthcoming paper for these facts.

\section{References}

[1] A. ANDreotTi AND Y-T. Siv, Projective embedding of pseudoconcave spaces, Ann. Scuola Norm. Sup. Pisa, 24 (1970), 231-278.

[2] A. FuJIKI, On automorphism groups of compact kähler manifolds, Invent. Math., 44 (1978), 225-258.

[3] K. Kodaira, On the structure of compact complex analytic surfaces, I, Amer. J. Math., 86 (1964), 751-798.

[4] H. TsuJI, On the neighborhood of a Hopf surface, preprint.

Present Address:

Department OF Mathematics

SOPHIA UNIVERSITY

Chiyoda-kU, Tokyo 102 